\title{
PIERO CALAMANDREI Y LA TUTELA CAUTELAR ${ }^{(*)}$
}

\author{
REMOCAPONI $^{(* *)}$
}

\begin{abstract}
Resumen
Se pondera la notable contribución de Piero Calamandrei al estudio la tutela cautelar en la obra "Introduzione allo studio sistematico dei provvedimenti cautelari", considerada un clásico de la literatura del derecho procesal civil. El autor reflexiona sobre la habilidad de síntesis y carácter sistemático del pensamiento de Calamandrei que incluso hoy, a través de la experiencia europea, constituye un modelo de referencia que ha contribuido de manera decisiva a la distinción de las medidas cautelares, que están dirigidas a garantizar la eficacia de la función jurisdiccional.
\end{abstract}

Palabras clave: Tutela jurisdiccional, proceso civil, medidas cautelares. Abstract
We remarkable the contribution of Piero Calamandrei, the study of the cautionary
tutela in the work: Introduzione allo studio sistematico del provvedimenti cautelari ",

(*) Título original en italiano: Piero Calamandrei e la tutela cautelare. Traducido al español por Yuri Tornero Cruzatt. Yuri Tornero es traductor del Centro de Estudios sobre América Latina de la Universidad de Bolonia. Miembro desde el 2013 de la "Associazione Italiana di Diritto Comparato". Cursa estudios de doctorado en Comparación Jurídica e Histórico Jurídica por la Università di Ferrara (Italia). Titulado de abogado en la Universidad Nacional Mayor de San Marcos. Hha realizado una estadía en el Max Planck Institute for European Legal History (MPleR) - Alemania.

(**) Remo CAPONI es profesor ordinario a tiempo completo de derecho procesal civil en la Universidad de Florencia desde el 2001. Ha enseñado Derecho de los contratos internacionales en la Universidad de Siena desde 1995 hasta el 2009. Ha enseñado Derecho procesal civil en la Universidad de Siena entre 1995 al 2000. Ha enseñado Introducción al derecho italiano en la Universidad de Bielefeld (República Federal Alemana) del 1989 al 1991. Becado de la Fundación 'Alexander von Humboldt de Bonn'. Ha colaborado con 'Foro italiano' del 1992 al 2013. Es miembro de 'International Journal of Procedural Law','Rivista di diritto processuale', 'Rivista trimestrale di diritto e procedura civile', 'Int'l Lis'. Associazioni scientifiche e culturali: Associazione italiana fra gli studiosi del processo civile (Consiglio direttivo), European Law Institute, ELI (Executive Committee), Accademia di diritto europeo, ERA (Comitato consultivo), Associazione internazionale di diritto processuale (Council), Wissenschaftliche Vereinigung für Internationales Verfah-rensrecht e. V., Deutsch-italienische Juristenvereinigung, Associazione italiana "Alexander von Humboldt", Centro interuniversitario di ricerca e di studi sulla giustizia civile "Giovanni Fabbrini" (Board of the Directors). 
which is considered a classic of the literature of procedural law". The author reflects on the ability of synthesis and systematic of Calamandrei thought., that even today through European experience, constitutes a reference model, which has contributed decisively in the distinction of precautionary measures which are aimed to ensure the effectiveness of the judicial fuction

Keywords: Judicial protection, civil process, precautionary measures.

\section{Sumario}

1.- Un clásico. 2.- Una genial intuición. 3.- El peligro de retraso. 4.- La teoría de Calamandrei en Europa. 5.- La teoría de Calamandrei y la dimensión colectiva, social de la justicia civil. 6.- La tutela cautelar y el Tribunal Constitucional. 7.- Piero Calamandrei y Alessandro Pekelis.

\section{Un clásico}

Llevo entre manos una edición original de la "Introduzione allo studio sistematico dei provvedimenti cautelari" de Piero Calamandrei ${ }^{1}$. El libro me había fascinado mientras estudiaba para mi tesis de licenciatura. En 1986 decidí invertir el primer plazo de mi beca de doctorado en comprar libros. En primer lugar en la lista de mis deseos había la colección "Opere giuridiche" de Piero Calamandrei cuidada por Mauro Cappelletti desde 1965 hasta 1984 con la editorial Morano de Nápoles. La encontré. Pero también tuve la suerte de encontrar, en la librería del anticuariado Gozzini en Florencia, una copia de 1936 del libro.

Normalmente se necesitan años para que una obra jurídica pueda considerarse "clásica"; eso solo después que se haya valorado su capacidad de influir sobre los estudios siguientes y de marcar un cambio decisivo en la práctica de tratar un solo tema durante un largo lapso temporal. Muy pocas son la obras que nacen como clásicas, que son clásicas en el mismo momento en que salen de la tipografía, por que este cambio se percibe al instante, tan solo con una rápida confrontación entre el contenido de estas obras y la literatura anterior. La Introduzione forma parte de las mismas. Cuando Piero Calamandrei la escribe, está en la cumbre de su carrera: un año antes publicó "Elogio dei giudici scritto da un avvocato", , otra obra que le dará mucha fama a lo largo del tiempo también gracias a sus traducciones en varios idiomas. Pero sobre todo, dieciséis años antes, él había publicado "La cassazione civile".

1 P. CALAMANDREI. Introduzione allo studio sistematico dei provvedimenti cautelari, Padua 1936.

2 P. CALAMANDREI. Elogio dei giudici scritto da un avvocato, Florencia 1935. 
Esta obra monumental, que permanece como una de las mejores de la literatura italiana en el ámbito del derecho procesal civil, le había procurado ya una importante notoriedad y una notable estima también en Alemania ${ }^{3}$.

\section{Una genial intuición}

En la "Introduzione allo studio sistematico dei provvedimenti cautelari" hay ante todo una lección por lo que concierne al método. Según lo que el mismo Calamandrei escribe al empezar la obra, el texto contiene la parte introductora "de un curso sobre los procedimientos cautelares dirigido (...) a los estudiantes del segundo año" de la Facultad de Derecho de Florencia ${ }^{4}$. En esta concretización del modelo humboltiano de Universidad, en que se encuentran la investigación y la enseñanza a un nivel máximo, puede tomarse hoy algo singular.

También hay un ejemplo de síntesis: 162 páginas, índices incluidos. Capacidad de tomar lo esencial sin dilatarse en detalles inútiles. Otra habilidad de la que se carece hoy en día.

La contribución de Piero Calamandrei no es la primera en la reflexión sobre la tutela cautelar. Antes tuvimos el ensayo de Agostino Diana de $1909^{5}$ y las importantes páginas que Chiovenda dedicó al tema, antes en "Principi di diritto processuale civile"6 y después en "Istituzioni di diritto processuale civile" . $\mathrm{El}$ autor relaciona las medidas cautelares con el principio que "el proceso tiene que garantizar, a quién tenga derecho y dentro de lo posible prácticamente, todo y justo lo que el mismo tiene derecho a conseguir" y

3 Cfr. E. SCHWINGE, Grundlagen des Revisionsrechts. Rechtsdogmatisch rechtsvergleichend rechtspolitisch, Bonn1935.

4 Calamandrei recordará que en el decenio entre 1924 y 1934, la Facultad de Derecho de Florencia, sirviendose de cierta flexibilidad de los ordenamientos didácticos que entonces se concedía a las universidades por la reforma Gentile, había dividido el cuatrienio de los estudios de derecho en dos bienios, el primero dedicado a la enseñanza en general de todas las disciplinas fundamentales, dada en forma de cursos introductorios con carácter informativo; el segundo dedicado a la enseñanza detallada de cada capítulo de las mismas disciplinas , o de disciplinas especificas incluidas en dichas enseñanzas, dada en forma de cursos monográficos complementados por ejercitaciones, con carácter predominantemente formativo [...]. De uno de estos cursos monográficos, creados día a día en la facultad a través de las discusiones con los mejores estudiantes, ha surgido, por ejemplo, mi obra sobre la Introduzione allo studio sistematico dei provvedimenti cautelari"; asì, Piero Calamandrei, Istituzioni di diritto processuale civile secondo il nuovo codice, advertencia a la primera edición, Padua 1941.

5 A. DIANA, Le misure conservative interinali, in Studi senesi, Turín 1909, 210 sgtes.

6 G. CHIOVENDA, Principi di diritto processuale civile, edición anastática de la tercera impresión, con prefacio de V.Andrioli, Nápoles 1980.

7 G. CHIOVENDA, Istituzioni di diritto processuale civile, Nápoles 1933. 
llega a considerar "la acción aseguradora" como práctica general, aunque en un sistema que conocía una disposición de contenido análoga al actual art. 700 c.p.c. (Código procesal civil). Con eso la opinión de Chiovenda revelaba, por decir así, su principal "defecto", o sea que "el dinamismo de su sistema requería por parte de los operadores prácticos y teóricos una capacidad de comprensión probablemente incobrable en las primeras décadas del siglo" ${ }^{8}$.

El "apremio de la legalidad" ${ }^{\prime}$, que de manera implícita es un elogio burgués de la jurisprudencia, impedirá que Calamandrei siga a Chiovenda en sus creaciones más atrevidas, por ejemplo en la acción aseguradora general considerada como instituto del derecho vigente (y además, fuera del campo de la tutela cautelar, como la acción naciente del contrato preliminar). De todas maneras es cierto que la obra del jurista florentino se rescata con una genial intuición de las constricciones de un análisis centrado en cada una de las medidas cautelares, para ofrecer una visión teórica general que, lo mismo que todas las concepciones auténticas y meditadas de esta natura, es a la vez puntualización del presente, individuación de las evoluciones sucesivas y además inicio de la concretización de semejantes tendencias. Es suficiente leer las últimas dos páginas del libro, las cuales casi constituyen un programa legislativo que luego efectivamente se realizó en sus características esenciales, como la introducción a un "poder cautelar general" y a una "tipología uniforme de procedimiento sumario cautelar" ${ }^{\prime 10}$.

\section{El peligro del retraso}

El carácter sistemático del pensamiento de Calamandrei incluso hoy constituye un modelo de referencia en la interpretación de la mayor novedad legislativa de los últimos veinte años en materia de tutela cautelar: la que fue introducida por la ley n. 80/2005 la cual, reuniendo propuestas formuladas hace tiempo en dicha materia y anticipadas en el proceso societario, ha interrumpido el nexo estructural entre medida cautelar y "causa de mérito"11 en relación a la cesión de una de las medidas cautelares enumeradas por el art. 669-octies, inciso $6^{\circ}$, c.p.c. (en breve: medidas cautelares "anticipatorias") dejando intacto ese nexo en relación a la cesión de medidas cautelares de contenido puramente "preventivo".

\footnotetext{
Así, A. ProtoPisanI, Chiovenda e la tutela cautelare, en Riv. Dir proc. 1988, 16 sgtes., esp. 34.

Así, P. Grossi, Stile fiorentino, Milán 1986, 146 sgtes., esp.150.

Cfr. P. CALAMANDREI, Introduzione allo studio sistematico dei provoedimenti cautelari, cit., 147.

1 La "causa di merito" se refiere a la causa en la cual se conoce tanto el derecho como los hechos. (N. del T.)
} 
En efecto, favorece la distinción entre las dos categorías de medidas; una reinterpretación, a través de la experiencia europea, de las páginas de Piero Calamandrei que han contribuido de manera decisiva en Italia a la elaboración de la distinción entre medidas cautelares conservativas y anticipativas $^{12}$.

De hecho, además de las medidas preventivas, en la experiencia europea pueden enumerarse tres tipologías de contenido por lo que se refiere a las medidas provisionales: las medidas que conservan la situación de hecho y de derecho con el objeto de asegurar la futura ejecución forzada; las medidas de reglamentación de una situación provisional; las medidas que anticipan el contenido de la disposición definitiva y que enseguida satisfacen, de manera parcial o total, el interés protegido por el derecho deducido en juicio ${ }^{13}$.

Calamandrei distingue medidas sumariales anticipadas, medidas dirigidas a asegurar la ejecución forzada, medidas dirigidas a adelantar las disposiciones decisorias, caución procesal $^{14}$. Aparte de las cauciones procesales que son una tipología de medidas dirigidas a asegurar la ejecución forzada (de la condena a la indemnización de los daños producidos por la ejecución de una medida cautelar que resulta ser infundada), y las medidas preventivas, muy importante es la bipartición entre medidas preventivas (de la eficacia práctica de la ejecución forzada) y de las medidas anticipatorias (de las medidas decisorias).

En la opinión de Calamandrei, la categoría de medidas preventivas incluye las medidas provisionales pertenecientes al segundo y al tercer tipo de la actual experiencia europea (o sea las medidas disciplinarias de una situación provisional y las medidas anticipatorias del contenido de la disposición definitiva). Esta impostación se explica muy simplemente considerando que el jurista escribe su obra sobre la tutela cautelar cuando las medidas de la tercera tipología todavía no habían obtenido una marcada elaboración conceptual más formaban parte de la categoría de las medidas de regulación

12 Para un discurso más amplio sobre este punto, v. R. CAPONI, La nuova disciplina dei procedimenti cautelari in generale (t. n. 80 de 2005), en Foro it. 2006, V, c. 69.

13 Cfr. R. STÜRNER, Einstweilinger Rechtsschutz, General Bericht, presentado en ocasión del coloquio de Bruxelles el 26 y 27 de octubre de 2001 por iniciativa de la Associación internacional de derecho procesal y del Centro interuniversitario belga de derecho procesal, ahora en M. Storme (a cargo de), Procedural Laws en Europe - Towards Harmonisation, Antwerpen/Apeldoorn 2003, 143 sgtes.

14 Cfr. P. CALAMANDREI, Introduzione allo studio sistematico dei provvedimenti cautelari, cit., 31 sgtes. 
de una situación provisional, en particular según las características que éstos asumen en el ordenamiento alemán a través del § 940 Zpo.

Calamandrei escribe: "forman parte de ese [...] conjunto medidas con las que se establece temporalmente, a la espera de que a través del proceso ordinario sea formulada la decisión definitiva, una relación controvertida, de la incertidumbre de la cual, si esta perdurara hasta la emanación de la disposición definitiva, podrían derivar daños irreparables por una de las partes $^{15}$. Entre los casos típicos de esta categoría él incluye las denuncias de obra nueva y de daño eventual, las medidas provisionales y urgentes en el interés de los cónyuges y de la prole en el proceso de separación personal, la pensión alimenticia provisional, el secuestro de las cosas que un deudor ofrece por su liberación. En ese grupo "la medida cautelar consiste precisamente en una decisión anticipada y provisional del mérito, destinada a durar hasta que a esta regulación provisional de la relación controvertida se sobreponga una regulación conseguida de manera estable a través del más lento proceso ordinario" ${ }^{16}$.

Calamandrei individua un rasgo funcional común a las medidas incluidas en ese grupo, o sea que estas son dirigidas a neutralizar el peligro de retraso de la resolución definitiva ${ }^{17}$. Diferente pero igualmente merecedor de neutralización con respecto al peligro de infructuosidad práctica (conforme más bien a la ejecución forzosa). De tal manera, él favorece un notable avance en la teoría de las medidas cautelares en la experiencia italiana en oposición a la experiencia alemana, por entonces todavía atrapada en una visión de la tutela cautelar como esencialmente funcional a la utilidad en la práctica de la ejecución forzosa ${ }^{18}$. Dicho avance consiste en liberarse de la idea que el contenido que anticipa la disposición sea un rasgo excepcional de la tutela cautelar: una adquisición difícil por parte de la doctrina alemana, la cual a esta teoría se abre solo al final de los años sesenta del siglo $X X$, empezando

\footnotetext{
Así, P. CALAMANDREI, Introduzione allo studio sistematico dei provvedimenti cautelari, cit., 38. Así, P. CALAMANDREI, Introduzione allo studio sistematico dei provvedimenti cautelari, cit,, 38 sgtes. Así, P. CALAMANDREI, Introduzione allo studio sistematico dei provvedimenti cautelari, cit., 56 sgtes.

18 Recordamos que en el ordenamiento alemán la disciplina de las medidas cautelares està incluída en la sección quinta del octavo libro de la Zivilprozeßordnung, dedicado a la ejecución forzosa.
} 
por los estudios, aunque diversamente dirigidos, de Fritz Baur ${ }^{19}$ y de Dicter Leipold ${ }^{20}$.

De esta manera la teoría de Calamandrei permite sostener eficazmente la conclusión que, con excepción del caso del secuestro, las demás medidas cautelares entran en la categoría de los procedimientos preventivos y, así por consiguiente, en la limitación del principio de instrumentalidad.

\section{La teoría de Calamandrei en Europa}

La idea de Calamandrei se abre eco también en Europa en un caso que se ha quedado sin solucionar por parte del tribunal de justicia y que es de los más sensacionales "por los protagonistas implicados y por la institución puesta en tela de juicio: el máximo órgano judicial inglés de entonces, la House of Lords, se pregunta si el principio, profundamente arraigado en el derecho constitucional aún antes que en el derecho procesal británico, según el cual al juez no se permite orientar interlocutory injunctions con finalidad cautelar en contra del gobierno" ${ }^{21}$ (más rigurosamente, en contra de la Corona: the King can do no wrong), pueda oponerse al derecho comunitario. El Tribunal establece que "la completa eficacia del derecho comunitario se encontraría reducida si una norma de derecho nacional impidiera al juez, llamado a dirimir una controversia disciplinada por el derecho comunitario, conceder medidas provisionales con el objetivo de garantizar la plena eficacia del pronunciamiento jurisdiccional sobre la existencia de los derechos invocados en apoyo al derecho comunitario". "En tal situación, el juez no puede aplicar la norma de derecho nacional que impida la concesión de medidas provisionales $^{\prime 22}$. Esta perspectiva se desvela en las conclusiones, encargadas al abogado General Giuseppe Tesauro, las cuales aclaran de forma ejemplar la conexión entre la efectividad de la tutela jurisdiccional y las medidas cautelares con referencia a la doctrina clásica italiana, en el párrafo 12 de Istituzioni di diritto processuale civile di Giuseppe Chiovenda y en la Introduzione di Piero Calamandrei. Pero incluso antes el Abogado General esta es una pequeña anécdota - va de exploración: trae desde Italia la obra de Piero Calamandrei y encarga la traducción al inglés de sus largas partes al servicio de traducción y interpretación del Tribunal de Justicia y hace que la

19 F. BAUR, Studien zum einstweilingen Rechtsschutzes, Tübingen 1967.

20 D. LEIPOLD, Grundlagen des einstweilingen Rechtsschutzes im zivil-, verfassungs- und verwaltungsgerichtlichen Verfahren, Mónaco 1971.

21 N. TROCKER, Il diritto processuale europeo e le "tecniche" della sua formazione: l'opera della Corte di giustizia, en Europa e diritto privato 2010, 361 sgtes., esp. 388 sgtes.

22 Cfr. Tribunal de Justicia CE19 dejunio 1990, C-213/89, Factortame. 
traducción sea repartida entre los jueces ${ }^{23}$. El "arte mágico de escribir" de Piero Calamandrei puesto en evidencia por Franco Cipriani ${ }^{24}$ (en realidad se trata de una calidad común a los dos) ha logrado de esta manera difundir su teoría en Europa.

\section{La teoría de Calamandrei y la dimensión colectiva, social de la justicia civil}

En una página que podemos definir dura por su contenido, Calamandrei sostiene que las medidas cautelares son dirigidas "más que a defender los derechos subjetivos, a garantizar la eficacia y, por decir así, la seriedad de la función jurisdicional [...]. Las medidas cautelares están preparadas, más que en el interés del individuo, en el interés de la administración de la justicia, de la que garantizan el buen funcionamiento y además el buen nombre, que digamos. Si la expresión "policía judicial" no tuviera ya en nuestro ordenamiento un significado preciso, esta podría parecer apta a denotar la tutela cautelar ${ }^{\prime 25}$. Dicha página no ha podido escapar a la crítica de Franco Cipriani $^{26}$. No puede evitarse recordar esta página, al menos por incluirla eventualmente en una época ya superada, la de una exasperada orientación hacia el derecho público de la doctrina del proceso. Pero esta sería la interpretación más simple.

Este pasaje demuestra que la teoría de Calamandrei siempre ha sido orientada a captar la dimensión colectiva, social de los problemas de la justicia civil, incluso tratando de las instituciones más técnicas. Desde este punto de vista, el enfoque de Calamandrei no resulta para nada superado, más bien está a la vanguardia, ya que capta la oportunidad de conformar la doctrina del proceso civil en función de importantes objetivos de política pública, nuevos con respecto a la tutela jurisdiccional de los derechos individuales deducidos en juicio. De otra manera no se comprendería la evolución del derecho de la Unión europea, desde que la doctrina del proceso civil ha sido explícitamente conectada con la realización del mercado interno ${ }^{27}$ y, más recientemente (con el Tratado de Amsterdam de 1997), con

23 Se debe la citación de este episodio a la cortesía del prof. Giuseppe Tesauro.

24 F. CIPRIANI, Piero Calamandrei e la procedura civile, 2a. edición revisada y ampliada, Nápoles 2009, 11.

25 P. CALAMANDREI, Introduzione allo studio sistematico dei provvedimenti cautelari, cit., 143 sgtes.

26 F. CIPRIANI en nota a la reedición de la recensión de V. Andrioli, Sull' "Introduzione allo studio sistematico dei provvedimenti cautelari" di Piero Calamandrei, en Foro it.2009, V. 205.

27 El impulso fundamental hacia este enlace historico viene de la jurisprudencia del Tribunal de Justicia, en particular desde Trib. de Just. CE 10 de febrero 1994, C-398/92, Mund E Fester. 
la creación gradual de un "espacio de libertad, seguridad y justicia". Si no, no se entenderían las orientaciones que, si no se considera la historia de la justicia laboral, se destacan con evidencia en el ordenamiento italiano sobre todo a partir de la 1. n. 80/2005, incluyendo las reformas de la justicia civil en los campos de acción por el desarrollo económico, social y territorial del país.

\section{La tutela cautelar y el Tribunal Constitucional}

Es superfluo señalar las etapas del éxito que la tutela cautelar, gracias al estudio de Piero Calamandrei, ha conocido en el ordenamiento italiano. En cambio es necesario recordar que todavía hay una frontera por atravesar: la emisión de medidas cautelares por parte del Tribunal Constitucional. En el ordenamiento alemán, algunos de los más importantes procedimientos delante del Bundesverfassungsgericht han conocido un pronunciamiento sobre una instancia cautelar, en particular de suspensión de la ley la cual constitucionalidad es puesta en tela de juicio. Reconocer que la aceptación de la instancia es una excepción, en el mismo modo en que ocurre en el ordenamiento alemán, es algo. Rechazar la idea que esto sea compatible con el papel del Tribunal Constitucional en el sistema, como se propende a afirmar en el ordenamiento italiano, es otra cosa. Esto esconde la escasa capacidad de familiarizar con este instrumento y es producto de una insuficiente habilidad de argumentación ${ }^{28}$. En particular, cuando se trata de leyes que requieren un empeño y un esfuerzo de organización notables, no puede evitarse que el peso de los hechos consumados antes del pronunciamiento del Tribunal, que no pueden ser eliminados (mejor dicho, pueden eliminarse, pero con mucha dificultad), influya en el balance constitucional y juegue un papel fundamental en favor de los argumentos útiles para salvaguardar la ley cuya constitucionalidad se duda. Por una parte puede aceptarse esta situación inaceptable y por otra parte puede evaluarse seriamente la alternativa de la suspensión cautelar erga omnes de la eficacia de la ley. Tertium non datur. Pensemos, por ejemplo, en el juicio de constitucionalidad de la ley reciente que ha introducido la tentativa obligatoria de mediación por un número notable de conflictos (d.lgs. $n$. 28/2010).

28 Sobre el tema, ver A. GRAGNANI, La cognizione cautelare nel processo costituzionale: L'esperienza del tribunale costituzionale federale tedesco, en Riv. dir. Cost. 2005, 157 sgtes. 


\section{Piero Calamandrei y Alessandro Pekelis}

Antes de terminar ese tema, han suscitado atención en mí las últimas líneas de la advertencia inicial de la Introduzione: “(...) que mi esperanza no será decepcionada, se demostrará en esta misma Collezione por el volumen, ya anunciado, sobre la teoría de las medidas cautelares, gracias al ingenio agudo de Alessandro Pekelis" ${ }^{\prime 29}$. Dicho libro nunca fue escrito: dos años después se aprobarán en Italia las infames leyes raciales y Alessandro Pekelis, hebreo ruso de Odessa llegado a Italia al principio de los años veinte, será obligado a refugiarse en los Estados Unidos con su familia. Me puse a seguir otros rastros de material documental, como quizás habría hecho Franco Cipriani, en busca de contactos sucesivos entre Piero Calamandrei y Alessandro Pekelis en aquel periodo tan difícil. Mi búsqueda ha sido premiada. En una página del libro de memorias de Carla Pekelis ${ }^{30}$, mujer de Alessandro, se cuenta que en torno al final del año 1941 la familia Pekelis se encontraba en Lisboa, a la espera de que obtuvieran los visados para emigrar a Estados Unidos. Las autoridades estadounidenses titubeaban: esperaban una prueba de la identidad política de Alessandro Pekelis y esa prueba llegó gracias a la ayuda de Piero Calamandrei: "cuando telegrafiamos a Piero Calamandrei, profesor en la Universidad de Florencia y amigo muy estimado, pidiéndole un recorte del artículo" (de un periódico fascista a través del cual se criticaba personalmente a Alessandro Pekelis) "lo hicimos con cierta perplejidad. ¿Nuestra petición podía procurarle problemas? (...). el recorte del artículo llegó muy rápido (...). ¡Dios te bendiga, Calamandrei! El artículo fue decisivo para alcanzar el objetivo. El día 20 de noviembre, después de una ceremonia que duró cinco horas, nos concedieron oficialmente los visados americanos".

29 P. CALAMANDREI, Introduzione allo studio sistematico dei provoedimenti cautelari, cit., VIII.

30 C. PEKELIS, La mia versione dei fatti, Palermo 1996, 167 sgtes. 